\title{
The impact of one HF-rTMS session on fine motor function in right-handed healthy female subjects: a comparison of stimulation over the left versus the right dorsolateral prefrontal cortex
}

\author{
Baeken C. ${ }^{1,2}$ CA* $^{*}$, Schrijvers D.L. ${ }^{3,5^{*}}$, Sabbe B.G.C. ${ }^{3,5}$, Vanderhasselt M.A. ${ }^{4}$ and De Raedt R. ${ }^{4}$ \\ (1) Department of Psychiatry, University Hospital (UZBrussel), Brussels, Belgium \\ (2) Center for Neurosciences, Vrije Universiteit Brussel (VUB), Brussels, Belgium \\ (3) Collaborative Antwerp Psychiatric Research Institute, University of Antwerp, Faculty of Medicine, Antwerp, \\ Belgium \\ (4) Department of Experimental, Clinical \& Health Psychology, Ghent University, Ghent, Belgium \\ (5) Psychiatric Hospital Sint-Norbertushuis, Duffel, Belgium \\ ${ }^{\text {CA }}$ Corresponding author: Chris Baeken, M.D., Ph.D.Department of Psychiatry, University Hospital \\ (UZBrussel),Vrije Universiteit Brussel (VUB). Laarbeeklaan 101, 1090 Brussels, Belgium. Tel: \\ +3224776425. Fax: +3224776800. E-mail: chris.baeken@uzbrussel.be \\ * These authors contributed equally to this work.
}

Keywords: HF-rTMS; DLPFC; lateralization; fine motor functioning; volunteers 


\section{Abstract:}

Background: Repetitive transcranial magnetic stimulation (rTMS) is a non-invasive tool to investigate neural conduction in motor processes. Most rTMS research has been conducted by targeting the primary motor cortex. Several studies found also increased psychomotor speed after rTMS of the dorsolateral prefrontal cortex (DLPFC). However, these studies were mainly performed in psychiatric patients, only targeting the left DLPFC, and often without sham control. Moreover, psychomotor speed is mostly measured based on tasks that also require higher executive functions.

Methods: Here, we examined the lateralized effect of one sham-controlled High Frequency (HF)-rTMS session applied to the left or right DLPFC on fine motor function in thirty-six healthy right-handed females, using the Fitts‘ paradigm.

Results: We found a significant improvement in psychomotor speed only after actively stimulating the right DLPFC.

Conclusion: Our results support the assumption of a right prefrontal neural network implicated in visuomotor behaviour and performance processes, and that the improvement in psychomotor speed is not a secondary effect of decreased mood. 


\section{Introduction}

Transcranial Magnetic Stimulation (TMS) is a non-invasive technique used to investigate neural conduction. Depending on brain location and parameters of its delivery it can either activate or suppress motor, sensory, or cognitive functions [1-4]. In single TMS and repetitive TMS (rTMS) studies, these neurophysiological effects are not only dependent of the frequency used, but the net effects are also related to hemispheric lateralization differences [5-7].

Most studies on healthy subjects examined the impact of TMS on motor functioning by targeting the primary motor cortex [8-11]. In contrast to most other cortical areas, stimulating the motor cortex results in immediate behavioural effects and these motor responses are relatively easy to detect visually or by motor evoked potentials (MEP) [11]. Besides the high or low frequency effects on motor responses, rTMS exerts different effects dependent on which hemispheric side of the motor cortex is stimulated [12].

The dorsolateral prefrontal cortex (DLPFC) is implied in complex motor planning, cognitive control and goal maintenance. It plays an important role in cognitive regulation, behavioural action control and working memory. The relationship of the DLPFC with executive-control adjustment suggests a pivotal in the dynamic tuning of executive control and, consequently, in behavioural adaptation, within a neurocircuitry including motor areas, anterior cingulate cortex (ACC) and subcortical circuits [13]. In line with this functional specialization, many studies have shown that rTMS of the DLPFC has marked effects on higher cortical functions such as attentional control, both in patients $[14,15]$ and healthy volunteers $[16,17]$. Recent clinical studies in majorly depressed patients showed a positive impact of high frequency (HF)-rTMS treatment on psychomotor symptoms by targeting the left DLPFC [18, 19]. Importantly, studies in patients (e.g. depression, schizophrenia) using experimental tasks found also increased psychomotor speed after rTMS of the DLPFC [4].

A first problem with these findings is that psychomotor speed is mostly measured based on tasks that also require higher executive functions [4]. A second problem is that the improvement in psychomotor speed might be a secondary effect of biological changes and decreased symptoms. Gender differences have also been reported, with improvements observed only in woman [20]. Moreover, most patient studies only applied rTMS to the left DLPFC, often without a sham control. In order to investigate the possible role of the DLPFC in pure psychomotor functioning, a straightforward simple fine motor task should be used before and after rTMS of the left and right DLPFC, using a sham controlled design, in a uniform sample of healthy volunteers. It is intriguing that, to our best knowledge, there are currently no such studies.

Therefore, we wanted to further examine the lateralized effect of one HF-rTMS session applied to the left versus right DLPFC on fine motor function in right-handed healthy subjects. To reduce gender and age variability into the data only young healthy females in the same age range were selected. Two different but comparable groups of healthy women were studied receiving one suprathreshold sham-controlled HF-rTMS session applied to the left or right DLPFC. Effects on psychomotor speed were assessed applying the Fitts' task [21], a rater-independent fine motor task requiring precise sensori-motor programming, initiation and execution of the muscle commands [22-24]. All participants also completed a Dutch version of the Profile of Mood States (POMS [25]) just before ( $\left.T_{1}\right)$ and just after each HF-rTMS session $\left(T_{2}\right)$ to exclude interference of possible mood changes on the Fitts' task.Because this is the first study in healthy individuals examining the effect of one such a HF-rTMS session delivered to the dorsolateral prefrontal cortices, we hypothesized that compared to sham one real stimulation session would improve psychomotor speed regardless of lateralization. 


\section{Methods}

In accordance with the guidelines laid down in the declaration of Helsinki, the study was approved by our ethics committee (UZBrussel). All subjects gave written informed consent and were financially compensated. This study was part of a larger project investigating the effects of HF-rTMS on cognitive functions.

For this 'single' blind sham-controlled crossover HF-rTMS study, in total thirty-six right-handed neverdepressed female students were recruited (mean Beck Depression Inventory [26] score $=2.05 \pm 2.03$ ). Before inclusion in the study, psychiatric disorders were excluded using the Mini-International Neuropsychiatric Interview [27]. Exclusion criteria were current or past history of epilepsy, neurosurgical interventions, having metal or magnetic objects in the brain, and being pregnant. A trained neurologist screened the MRI's also used for localization purposes for possible neurological disorders. No drugs were allowed, except birth-control pills. Right-handedness was assessed with the Van Strien Questionnaire [28].

The female volunteers were randomized into two groups. One group received one sham-controlled HFrTMS over the left DLPFC ( $\mathrm{n}=18$, mean age $=21.20 \pm 1.44$ years) and the other received one sham controlled HFrTMS over the right DLPFC $(n=18$, mean age $=24.50 \pm 2.49$ years $)$. For both groups we used a counterbalanced cross-over design. If a given volunteer received first an active session, the second session was sham. The volunteer receiving initially a sham session received active HF-rTMS the second time. To avoid carry-over effects from the previous stimulation, the second session was planned after a one week interval. HF-rTMS (10 $\mathrm{Hz}$ ) was applied at $110 \%$ MT (1560 pulses), by using a figure-of-eight-shaped double 70mm coil (Magstim Company Limited, Wales, UK). For the sham condition, the coil was held at an angle of $90^{\circ}$. In order to accurately target the left and the right DLPFC (Brodmann area 9/46), taking into account individual anatomical brain differences, the precise stimulation site and position of the coil was determined using MRI non-stereotactic guidance (see also [29]). Perpendicular to this point the precise stimulation site on the skull was marked and stimulated. The study was conducted conform the current safety guidelines [30].

All participants were asked to complete a Dutch version of the POMS [25], a 32-item inventory that assesses five mood dimensions: 'depression' ( 8 items: minimum score 0 ; maximum score 32 ), 'fatigue' (6 items: minimum score 0 ; maximum score 24), 'tension' (6 items: minimum score 0 ; maximum score 24$)$, 'anger' (7 items: minimum score 0; maximum score 28) and 'vigor' (5 items: minimum score 0; maximum score 20). Ratings were made on four-point scales: a rating of 0 indicates a low level and a rating of 4 indicating a high level for a given mood. These tests were taken just before $\left(T_{1}\right)$ and just after $\left(T_{2}\right)$ each stimulation session.

Just before and after stimulation, participants performed the Fitts' task (Figure 1A; for a detailed description see [31]). They received 2 trials consisting each of six pairs of circles to be connected. Participants performed the Fitts' task on sheets of paper placed on a digitizer (WACOM1218RE) in front of them, using a pressure-sensitive inking ballpoint pen. Subjects had to connect two vertically placed circles, depicted on the sheet of paper, by drawing a line of about one $\mathrm{cm}$. They were instructed to start in the middle of the top circle and to end in the middle of the lower circle, and to draw as quickly as possible. The accuracy of movement was varied by changing the circle diameter from $0.50 \mathrm{~cm}$ (see a) in trial 1 to $0.25 \mathrm{~cm}$ (see b) in trial 2. Movement time (MT) was recorded, i.e. the time between the start and the completion of each separate line drawing movement. The movement times of inaccurate line drawings, i.e. when the line was drawn from the lower to the 
upper circle and/or when the start/endpoint of the connecting line was situated outside the circle diameter, were not inserted in the analyses.

All results were analysed using the SPSS for Windows 16.0 software package. Statistical analyses were performed using mixed (M)ANOVA's. The significance level was set at $\mathrm{p} \leq 0.05$ for all analyses. First, to examine whether possible mood changes by the rTMS application could influence our results, mood changes were analyzed with a mixed 2X2X2 MANOVA. Within-subject factors were Session (pre vs. post stimulation) and Stimulation (active vs. Sham). The between-subjects factor was Group (left vs. right stimulation). The five POMS mood scales were the multiple dependent variables.

To evaluate whether one sham-controlled HF-rTMS session influences psychomotor speed we performed a 2X2X2 ANOVA with Session (pre vs. post stimulation) and Stimulation (real vs. sham) as withinsubject factor and Group (left vs. right stimulation) as between-subject factor. Mean movement time (MT) on the Fitts' task was the dependent variable. 


\section{Results}

\subsection{Mood results}

For an overview for all POMS subscales values see Table 1.

The MANOVA showed a significant main effect for Session $(F(5,30)=4.25, p<.01)$, a trend towards significance for Group $(F(5,30)=2.45, p=.06)$, but not for Stimulation $(F(5,30)=1.92, p=.12)$. Also the interaction effects between Session and Group $(F(5,30)=1.54, p=.21)$, between Stimulation and Group $(F(5$, $30)=.42, p=.83)$, and between Session and Stimulation $(F(5,30)=.65, p=.66)$ did not reach significance. Importantly, The crucial interaction effect between Stimulation, Session and Group was not significant $(F(5$, $30)=1.46, p=.23)^{1}$

Therefore, it can be concluded that no significant mood changes occur after neither left nor right dorsolateral prefrontal cortical HF-rTMS.

\subsection{Fitts' task}

See also Table 2. Because MT values were not normally distributed, square root transformation was used for data normalization. Mean MT values were analyzed using SPSS 16.0 (significance level $\mathrm{p} \leq 0.05$ ). An

The 2X2X2 ANOVA with Stimulation (real vs. sham) and Session (pre vs. post stimulation) as withinsubject factor and Group (left vs. right stimulation) as between-subject factor showed no significant main effect for Stimulation $(F(1,34)<.01, p=.93)$ or for Group $(F(1,34)=.06, p=.82)$. We observed a significant main effect for Session $(F(1,34)=18.47, p<.01)$. The interaction effects between Session and Stimulation $(F(1,34)=2.03$, $p=.16)$, between Stimulation and Group $(F(1,34)=.29, p=.59)$ and between Session and Group $(F(1,34)=1.87$, $p=.18)$ were not significant. However, the crucial three-way interaction effect was significant $(F(1,34)=4.33$, $p=.045)$. To follow-up this interaction effect, separate ANOVA's for each group were conducted

The ANOVA for the left-sided stimulated group showed no main effect for Stimulation $(F(1,17)=.16$, $p=.70)$. Although the main effect for Session was significant $(F(1,17)=7.64, p=.01)$, the crucial interaction effect between Stimulation and Session was not $(F(1,17)=.36, p=.56)$.

The ANOVA for the right-sided stimulated groupshowed again no main effect for Stimulation $(F(1,17)=.14, p=.71)$ but the main effect for Session was significant $(F(1,17)=11.16, p<.01)$. Here, the interaction effect between Stimulation and Session reached significance $(F(1,17)=4.41, p=.05)$. No active versus sham stimulation MT differences were observed before stimulation $(t(17)=.89, p=.39)$. Paired $t$-tests revealed a significant improvement of MT only after real HF-rTMS $(t(17)=3.38, p<.01)$, but not after sham $(t(17)=.86$, $p=.36)$.

\footnotetext{
${ }^{1}$ To ensure that the absence of mood effects would not be related to power problems, we also performed separate paired tests (pre vs. post stimulation) in the real and sham stimulation condition for both groups separately and for each POMS subscale. No significant effects on mood were found (all p's > .05)
} 


\section{Discussion}

We investigated the lateralized effect of one HF-rTMS session applied to the left versus right DLPFC on fine motor function in right-handed healthy female subjects, using the Fitts' task, which is designed to evaluate sensori-motor programming, initiation and execution of the muscle commands, not requiring higher order cognitive processes [31]. Firstly, one sham-controlled HF-rTMS session applied to the left or right DLPFC did not result in a subjectively aware mood change in our healthy female subjects, which means that mood changes cannot account for the effect.

Secondly, although stimulating the left DLPFC did not facilitate or inhibit fine motor performance,in essence our results follow the findings of Grosbras and Paus [32], who reported a decrease in reaction times predominantly after (single pulse) stimulation of the right frontal eye field (FEF) and not the left FEF, indicating that TMS over the right FEF facilitates visual detection resulting in reduced reaction times. It has to be noted that neuroanatomically the right DLPFC is closely connected with the right FEF (Brodmann area 8) [33]. Interestingly, Woolley and colleagues [34] reported that on a functional level the right visuomotor areas were integrated with somatosensory guidance to produce well-coordinated movements. Further, in a recent study, improved double pulse TMS induced motor response patterns were predominantly observed after stimulation of the right primary motor cortex [35]. Here, the authors suggested that the goal-directed motor behaviour was related to activation of the right anterior hemisphere.

In line with these assumptions, it has been stated that non-primary motor cortices are capable of influencing motor cortical output [36]. On the other hand, although the repetitive stimulation of the motor cortices in healthy individuals has revealed to influence motor performance, targeting the DLPFC did not always result in similar motor effects [37]. Other methodological issues besides the targeted area, such as subthreshold stimulation and a limited number of delivered pulses could, to some extent explain the discrepant results found in HF-rTMS motor performance paradigms [38, 39]. In our current study, we used a suprathreshold HF-rTMS design generating 1560 pulses per session which compares to a clinically more effective session performed in depressed patients [40]. The choice of more potent HF-rTMS parameters might result in enhanced facilitatory neuronal effects on surrounding cortical areas [41, 42]. However, without concomitant brain imaging techniques these assumptions should be interpreted cautiously at this point.

Because heterogeneous subject sampling such as age and gender could be an important methodological issue in TMS research, the choice of more 'homogeneous' group of young female right-handed subjects should be considered as a major advantage [43]. Albeit we used a more 'homogeneous' sample of young female subjects and targeted the DLPFC under 3D-MRI guidance, our study is limited in that the interpretation cannot be generalized to a broader population. Further, although we observed no effect of sham stimulation, tilting the coil at a $90^{\circ}$ angle could have lead to a partially active effect [44].

In conclusion, stimulating the right DLPFC with one suprathreshold HF-rTMS session influenced fine motor functioning in right-handed healthy females. Importantly, the observed effects on fine motor functioning cannot be attributed to possible induced mood changes. Our current observations further extend the role of the right DLPFC on fine motor functioning in healthy subjects. Because rTMS at a site distant from the motor cortex can change the excitability of circuits intrinsic to the motor cortex [45], our findings support the assumption of a right (dorsolateral) prefrontal neural network implicated in visuomotor behaviour and performance processes 
[46]. Future (r)TMS research should integrate brain imaging to further elucidate the neurobiological mechanisms implicated in right prefrontal cortical control on fine motor functioning.

\section{Acknowledgements}

This research was supported by a grant from the Scientific Fund W. Gepts UZBrussel.MAV (FWO08/PDO/168) is a postdoctoral fellow of the Research Foundation Flanders (FWO). The authors declare no conflict of interest.

\section{Financial Disclosures}

None declared 


\section{References}

1. Paus T, Castro-Alamancos MA, Petrides M: Cortico-cortical connectivity of the human middorsolateral frontal cortex and its modulation by repetitive transcranial magnetic stimulation. Eur. J. Neurosci 2001; 14: 1405-1411.

2. George MS, Nahas Z, Kozol FA, Li X, Yamanaka K, Mishory A, Bohning DE: Mechanisms and the current state of transcranial magnetic stimulation. CNS Spectr 2003; 8: 496-514.

3. Lee L, Siebner H, Bestmann S: Rapid modulation of distributed brain activity by Transcranial Magnetic Stimulation of human motor cortex. Behav Neurol 2006; 17:135-148.

4. Guse B, Falkai P, Wobrock T: Cognitive effects of high-frequency repetitive transcranial magnetic stimulation: a systematic review. J Neural Transm 2010; 117: 105-122.

5. Garcia-Toro M, Montes JM, Talavera JA: Functional cerebral asymmetry in affective disorders: new facts contributed by transcranial magnetic stimulation. J Affect Disord 2001; 66: 103-109.

6. Speer AM, Willis MW, Herscovitch P, Daube-Witherspoon M, Shelton JR, Benson BE, Post RM, Wassermann EM: Intensity-dependent regional cerebral blood flow during 1-Hz repetitive transcranial magnetic stimulation (rTMS) in healthy volunteers studied with $\mathrm{H} 215 \mathrm{O}$ positron emission tomography: I. Effects of primary motor cortex rTMS. Biol Psychiatry 2003; 54:818-825. a

7. Speer AM, Willis MW, Herscovitch P, Daube-Witherspoon M, Shelton JR, Benson BE, Post RM, Wassermann EM: Intensity-dependent regional cerebral blood flow during 1-Hz repetitive transcranial magnetic stimulation (rTMS) in healthy volunteers studied with $\mathrm{H} 215 \mathrm{O}$ positron emission tomography: II. Effects of prefrontal cortex rTMS. Biol Psychiatry 2003; 54:826-832. b

8. Garry MI, Kamen G, Nordstrom MA: Hemispheric differences in the relationship between corticomotor excitability changes following a fine-motor task and motor learning. J Neurophysiol 2004; 91:15701578.

9. Fitzgerald PB, Fountain S, Daskalakis ZJ: A comprehensive review of the effects of rTMS on motor cortical excitability and inhibition. Clin Neurophysiol 2006; 117:2584-2596.

10. Di Lazzaro V, Profice P, Pilato F, Dileone M, Oliviero A, Ziemann U: The effects of motor cortex rTMS on corticospinal descending activity. Clin Neurophysiol 2010; 121:464-473.

11. Jung SH, Shin JE, Jeong YS, Shin HI: Changes in motor cortical excitability induced by high-frequency repetitive transcranial magnetic stimulation of different stimulation durations. Clin Neurophysiol 2008; 119:71-79.

12. Gorsler A, Bäumer T, Weiller C, Münchau A, Liepert J: Interhemispheric effects of high and low frequency rTMS in healthy humans. Clin Neurophysiol 2003; 114:1800-1807.

13. Mansouri FA, Tanaka K \& Buckley M: Conflict-induced behavioural adjustement: a clue to the executive functions of the prefrontal cortex, Nature Reviews Neuroscience 2009; 10: 141-152.

14. Vanderhasselt MA, De Raedt R, Baeken C, Leyman L, Clerinx P \& D'haenen H: The influence of rTMS over the right dorsolateral prefrontal cortex on top-down attentional processes. Brain Research 2007; 1137, 111-116. 
15. Leyman L, De Raedt R, Vanderhasselt MA \& Baeken C: Effects of repetitive transcranial magnetic stimulation of the dorsolateral prefrontal cortex on the attentional processing of emotional information in major depression: A pilot study. Psychiatry Research 2011; 185: 102-107.

16. Leyman L, De Raedt R, Vanderhasselt M A \& Baeken C: Influence of high-frequency repetitive transcranial magnetic stimulation over the dorsolateral prefrontal cortex on the inhibition of emotional information in healthy volunteers. Psychological Medicine 2009; 39: 1019-1028.

17. De Raedt R, Leyman L, Baeken C, Van Schuerbeek P, Luypaert R, Vanderhasselt MA \& Dannlowski U: Neurocognitive effects of Hf-rTMS over the dorsolateral prefrontal cortex on the attentional processing of emotional information in healthy woman: An event-related fMRI study. Biological Psychology 2010; 85: 487-495.

18. Hoeppner J, Padberg F, Domes G, Zinke A, Herpertz SC, Grossheinrich N, Herwig U: Influence of repetitive transcranial magnetic stimulation on psychomotor symptoms in major depression. Eur Arch Psychiatry Clin Neurosci 2010; 260:197-202.

19. Baeken C, De Raedt R, Santermans L, Zeeuws D, Vanderhasselt MA, Meers M, Vanderbruggen N: HFrTMS treatment decreases psychomotor retardation in medication-resistant melancholic depression. Prog Neuropsychopharmacol Biol Psychiatry 2010; 34:684-687.

20. Huber TJ, Schneider U \& Rollnik J: Gender differences in the effect of repetitive transcranial magnetic stimulation in schizophrenia. Psychiatry Res 2003;120: 103-105

21. Pier MP, Hulstijn W, Sabbe BG: Differential patterns of psychomotor functioning in unmedicated melancholic and nonmelancholic depressed patients. J Psychiatr Res 2004; 38: 425-435.

22. Sabbe B, Hulstijn W, Van Hoof J, Zitman F: Fine motor retardation and depression. J Psychiatr Res 1996; 30:295-306.

23. Sabbe B, Hulstijn W, van Hoof J, Tuynman-Qua HG, Zitman F: Retardation in depression: assessment by means of simple motor tasks. J Affect Disord 1999; 55:39-44.

24. Sabbe B, Hulstijn W, Maes M, Pier M, Scharpé S, Zitman F: Psychomotor slowing, neuroendocrine responses, and behavioral changes after oral administration of meta-chlorophenylpiperazine in normal volunteers. Psychiatry Res 2001; 105:151-163.

25. Wald FD, Mellenbergh GJ: De verkorte versie van de Nederlandse vertaling van de Profile of Mood States (POMS). Nederlands tijdschrift voor de psychologie 1990; 45: 86-90.

26. Beck AT \& Steer RA:Internal consistencies of the original and revised Beck Depression Inventory. J Clin Psychol 1984; 40: 1365-1367.

27. Sheehan DV, Lecrubier Y, Sheehan KH, Amorim P, Janavs J, Weiller E, Hergueta T, Baker R, Dunbar GC: The Mini-International Neuropsychiatric Interview (M.I.N.I.): the development and validation of a structured diagnostic psychiatric interview for DSM-IV and ICD-10. J Clin Psychiatry 1998; 59: 22-33.

28. Van Strien JW. Handvoorkeur en taaldominantie. Neuropraxis 2001; 2: 10-15.

29. Peleman K, Van Schuerbeek P, Luypaert R, Stadnik T, De Raedt R, De Mey J, Bossuyt A, Baeken C: Using 3D-MRI to localize the dorsolateral prefrontal cortex in TMS research. World J Biol Psychiatry2010;11:425-430. 
30. Rossi S, Hallett M, Rossini PM, Pascual-Leone A and The Safety of TMS Consensus Group: Safety, ethical considerations, and application guidelines for the use of transcranial magnetic stimulation in clinical practice and research. Clin Neurophysiol 2009; 120:2008-2039.

31. Sabbe B, van Hoof J, Hulstijn W, Zitman F: Depressive retardation and treatment with fluoxetine: assessment of the motor component. J Affect Disord 1997; 43:53-61.

32. Grosbras M.-H., Paus T :Transcranial magnetic stimulation of the human frontal eye field: effects on visual perception and attention. Journal of Cognitive Neuroscience 2002; 14: 1109-1120.

33. Fuster JM: The prefrontal cortex--an update: time is of the essence. Neuron 2001; 30:319-333.

34. Woolley DG, Wenderoth N, Heuninckx S, Zhang X, Callaert D, Swinnen SP: Visual guidance modulates hemispheric asymmetries during an interlimb coordination task. Neuroimage 2010; 50:15661577.

35. Callaert DV, Vercauteren K, Peeters R, Tam F, Graham S, Swinnen SP, Sunaert S, Wenderoth N: Hemispheric asymmetries of motor versus nonmotor processes during (visuo)motor control. Hum Brain Mapp DOI: 10.1002/hbm.21110.

36. Chouinard PA, Paus T: The primary motor and premotor areas of the human cerebral cortex. Neuroscientist 2006; 12:143-52.

37. Pascual-Leone A, Tormos JM, Keenan J, Tarazona F, Cañete C, Catalá MD: Study and modulation of human cortical excitability with transcranial magnetic stimulation. J Clin Neurophysiol 1998; 15:333343.

38. Reis J, Swayne OB, Vandermeeren Y, Camus M, Dimyan MA, Harris-Love M, Perez MA, Ragert P, Rothwell JC, Cohen LG: Contribution of transcranial magnetic stimulation to the understanding of cortical mechanisms involved in motor control. J Physiol 2008; 586:325-351. a

39. Reis J, Robertson EM, Krakauer JW, Rothwell J, Marshall L, Gerloff C, Wassermann EM, PascualLeone A, Hummel F, Celnik PA, Classen J, Floel A, Ziemann U, Paulus W, Siebner HR, Born J, Cohen LG: Consensus: Can transcranial direct current stimulation and transcranial magnetic stimulation enhance motor learning and memory formation? Brain Stimul 2008; 1:363-369. b

40. Gershon AA, Dannon PN, Grunhaus L: Transcranial magnetic stimulation in the treatment of depression. Am J Psychiatry 2003; 160:835-845.

41. Maeda F, Keenan JP, Tormos JM, Topka H, Pascual-Leone A: Interindividual variability of the modulatory effects of repetitive transcranial magnetic stimulation on cortical excitability. Exp Brain Res 2000; 133:425-430.

42. Barrett J, Della-Maggiore V, Chouinard PA, Paus T: Mechanisms of action underlying the effect of repetitive transcranial magnetic stimulation on mood: behavioral and brain imaging studies. Neuropsychopharmacology. 2004; 29:1172-1189.

43. Martin JL, Barbanoj MJ, Schlaepfer TE, Thompson E, Perez V, Kulisevsky J: Repetitive transcranial magnetic stimulation for the treatment of depression. Systematic review and meta-analysis. $\mathrm{Br} \mathbf{J}$ Psychiatry 2003; 182: 480-491.

44. Loo CK, Taylor JL, Gandevia SC, McDarmont BN, Mitchell PB, Sachdev PS:Transcranial magnetic stimulation (TMS) in controlled treatment studies: are some "sham" forms active? Biol Psychiatry 2000; 47: 325-331. 
45. Münchau A, Bloem BR, Irlbacher K, Trimble MR, Rothwell JC: Functional connectivity of human premotor and motor cortex explored with repetitive transcranial magnetic stimulation. J Neurosci 2002; 22:554-561.

46. Hoshi E: Functional specialization within the dorsolateral prefrontal cortex: a review of anatomical and physiological studies of non-human primates. Neurosci Res 2006; 54:73-84. 
Figure and Table legends
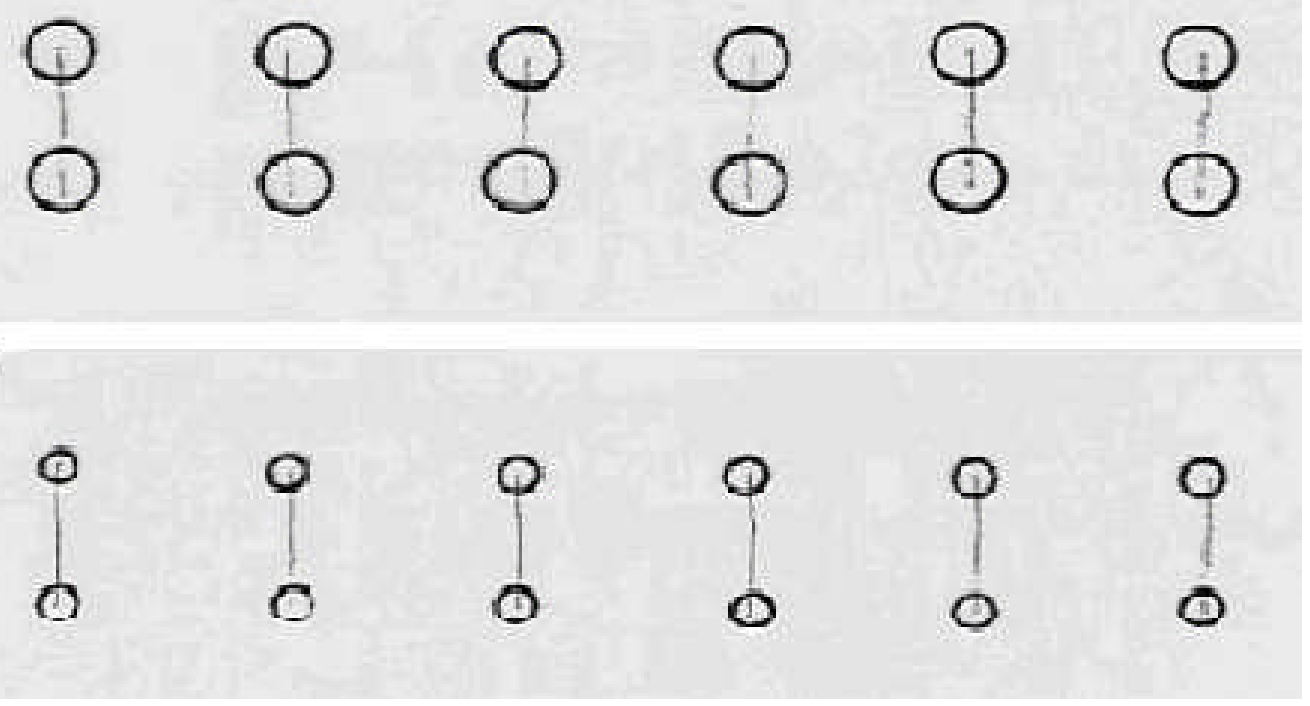

Fig 1. Visualization of the Fitts' task.

Participants performed the Fitts' task on sheets of paper placed on a digitizer (WACOM1218RE) in front of them, using a pressure-sensitive inking ballpoint pen. Subjects had to connect two vertically placed circles, depicted on a normal sheet of paper, by drawing a line of about one $\mathrm{cm}$. They were instructed to start in the middle of the top circle and to end in the middle of the lower circle, and to draw as quickly as possible. The accuracy of movement was varied by changing the circle diameter from $0.50 \mathrm{~cm}$ (see upper circles) in trial 1 to $0.25 \mathrm{~cm}$ (see lower circles) in trial 2. 


\begin{tabular}{|c|c|c|c|c|}
\hline & \multicolumn{2}{|c|}{ Active HF-rTMS } & \multicolumn{2}{|c|}{$\underline{\text { Sham HF-rTMS }}$} \\
\hline & $T 1$ & $T 2$ & $T 1$ & $T 2$ \\
\hline \multicolumn{5}{|l|}{ Left DLPFC } \\
\hline $\begin{array}{l}\text { POMS } \\
\text { Depression }\end{array}$ & $.80(2.07)$ &. $.25(.64)$ & $.80(1.96)$ & $.15(.49)$ \\
\hline POMS Anger & $1.10(1.94)$ & $1.20(3.00)$ & 1.75 (1.97) & $.85(1.50)$ \\
\hline POMS Tension & $1.25(2.02)$ & $.15(.49)$ & $1.30(2.54)$ & $.35(.93)$ \\
\hline POMS Fatigue & $4.40(4.62)$ & $3.25(2.94)$ & $3.70(4.38)$ & $2.50(3.10)$ \\
\hline POMS Vigor & $11.30(4.67)$ & $10.40(3.53)$ & $12.65(4.42)$ & $11.90(3.31)$ \\
\hline \multicolumn{5}{|l|}{ Right DLPFC } \\
\hline $\begin{array}{l}\text { POMS } \\
\text { Depression }\end{array}$ & $.35(.81)$ & $.50(1.15)$ & $.15(.49)$ & $.20(.70)$ \\
\hline POMS Anger & $.85(1.84)$ & $2.10(3.64)$ & $1.00(1.97)$ & $1.15(2.78)$ \\
\hline POMS Tension & $1.05(1.96)$ & $.85(1.42)$ & $1.15(1.63)$ & $.80(1.44)$ \\
\hline POMS Fatigue & $2.50(2.95)$ & $1.60(2.19)$ & $1.45(1.70)$ & $1.25(1.94)$ \\
\hline POMS Vigor & $9.60(4.99)$ & $8.75(4.33)$ & $10.95(3.95)$ & $11.10(3.88)$ \\
\hline
\end{tabular}

Table 1. Mean ratings and standard deviations for all POMS subscales before $\left(T_{1}\right)$, and immediately ( $\left.\mathrm{T}_{2}\right)$ after HF-rTMS (active stimulation or sham condition) on the left and right DLPFC. 


\begin{tabular}{|l|l|l|l|l|l|l|l|}
\hline \multicolumn{4}{|c|}{ Left HF-rTMS } & \multicolumn{3}{c|}{ Right HF-rTMS } \\
\hline \multicolumn{2}{|c|}{ Real HF-rTMS } & \multicolumn{2}{|c|}{ Sham HF-rTMS } & \multicolumn{2}{c|}{ Real HF-rTMS } & \multicolumn{2}{c|}{ Sham HF-rTMS } \\
\hline Before & After & Before & After & Before & \multicolumn{1}{|c|}{ After } & Before & After \\
\hline .157 & .149 & .155 & .144 & .172 & .140 & .163 & .155 \\
$(.033)$ & $(.030)$ & $(.039)$ & $(.043)$ & $(.050)$ & $(.054)$ & $(.058)$ & $(.055)$ \\
\hline
\end{tabular}

Table 2. Mean pre- and post active/sham stimulation psychomotor outcomes (with standard deviation) in msec for the Fitts' task (untransformed data). 